\title{
COMPARISON OF SEALING ABILITY OF EXPERIMENTAL TRICALCIUM PHOSPHATE CEMENT VERSUS BIODENTIN AND MINERAL TRIOXIDE AGGREGATE USED FOR FURCATION PERFORATION REPAIR
}

\author{
Mostafa M. El-Bialy ${ }^{*}$, Magdy M. Ali ${ }^{* *}$, Reham Hassan $^{* * *}$ and Engy M. Kataia ${ }^{* * * *}$
}

\begin{abstract}
The scope of this study is to compare the sealing capability of experimental tricalcium phosphate cement versus Biodentine and Mineral Trioxide Aggregate used for furcation perforation repair.

Methodology: Ninety six extracted human molars were perforated in furcation area and unsystematically divided into four groups relative to the furcation repair material into: experimental tricalcium phosphate $(n=30)$, Biodentine $(n=30), \operatorname{MTA}(n=30)$ while the fourth group of samples was left unrepaired as control $(n=6)$. Each group was further subdivided into three subgroups according the evaluation period as 1 week, 1 month and 3 months. Samples were subjected to dye penetration test and values calculated using a stereomicroscope with magnification (25X). Kruskal Wallis test was utilized to show the difference between more than two groups in non-related samples.
\end{abstract}

Results: Biodentine group showed statistically significant least dye absorbance; statistically there wasn't a significant difference between the Experimental material and MTA.

Conclusion: The experimental tricalcium phosphate provides an easy handling and quick setting of repair material with comparable sealing ability to MTA.

KEYWORDS: Biodentine, Tricalcium phosphate, Dye penetration, Furcation Perforation, MTA.

\section{INTRODUCTION}

Perforation can be defined as an artificial opening to connect both the root canal system and the surrounding tissues and supporting structures, which are either created by clinician during cavity preparation, or due to biological cause such as resorption or caries $^{(1)}$. Root perforation can be considered a frequent mishap of endodontic

\footnotetext{
* Assistant Researcher at Restorative and Dental Materials Research Department National Research Center.

** Dean, Professor of Endodontics, Faculty of Dentistry, Beni Suef University.

*** Head of Endodontic Department, Associate professor Faculty of Dentistry, Minia University.

**** Assistant Researcher Professor, Restorative and Dental Materials Department, National Research Centre, Cairo, Egypt
} 
treatment, which a clinician will encounter on a regular basis. For endodontically treated teeth, it occurs in approximately $2-12 \%{ }^{(2)}$. Such perforations compromise the outcome of treatment. In 1990, Torabinejad $\mathrm{M}$ has brought up Mineral Trioxide Aggregate. Since then, it has been chosen for all dentinal defects because of its biocompatibility and capacity for induction of precipitates of calcium phosphate at interface between periodontium and repairing bone tissue. Nevertheless, this material has some disadvantages like slow setting and difficulty of handeling ${ }^{(3)}$. Biodentine (Septodont, Saint Maur des Fosse's, France), also popular as 'dentine in a capsule', can be considered as a biocompatible and bioactive dentine substitute that does not have the disadvantages of Calcium hydroxide and Mineral trioxide. Hargreaves et al (2011) stated that Biodentine can help the dentist in achieving biomimetic mineralization without exceeding the depths of a carious cavity. Biodentine acts as Bioactive cement which has mechanical properties similar to those of dentin. Moreover, Biodentine acts in a bio compatible manner, having valuable effects on living cells ${ }^{(4)}$. Numerous studies have compared the sealing ability and the healing after perforation repair using MTA and Biodentine ${ }^{(5,6)}$.

Tricalcium phosphate cements contain a biodegradable ceramic. Because these cements are compatible with periodontal tissues, they are very successful in periodontal therapy. Moreover, Tricalcium phosphate can repair perforations when used with layers of epithelium, collagen, and bone, showing only little amount of inflammatory cells at the site of perforation ${ }^{(7)}$.

The purpose of this study was to assess the sealing ability of experimental tricalcium phosphate, Biodentine and MTA as furcation repair materials in molar teeth using dye penetration test.

Our Hypothesis is that the experimental perforation repair material will have a similar outcome to that of Biodentine and MTA.

\section{MATERIALS AND METHODS}

\section{Sample size calculation}

A total sample size of 96 samples (90 for experimental groups and 6 for control group) was enough to spot the effect size of 0.40 , a power (1- $\beta$ ) of $80 \%$ and at a significant level of $5 \%(\mathrm{p}<0.05)$, each experimental group would be represented by 30 teeth and ten teeth $(n=10)$ for each time period, while for control group it will be 2 teeth for each time period. G*Power software version 3.1.9.4. was utilized for calculation of sample size, Where; $\mathrm{S}$ the effect size, $\alpha=0.05, \beta=0.2$ and Power $=1-\beta=0.8$.

\section{Sample selection and preparation}

After the approval of this research protocol by the ethical committee at National Research Centre (Registration number 17-077). Ninetysix mandibular molar human teeth extracted due to periodontal reasons were provided by the Department of Oral Surgery, Faculty of Dentistry, Minia University.

Teeth were coated with a single layer of nail polish and access opening was prepared. Canal orifices were enlarged halfway through the root then sealed with Cavit cement (3M, ESPE, Germany) to prevent any leakage through the canal orifices ${ }^{(8)}$.

Furcation perforation was made in the center of the chamber floor by \# 2 long-shank round bur. The width of the perforation corresponds to the bur diameter, whereas perforation depends on thickness of dentin and cementum from floor of pulp chamber to the furcation area ${ }^{(9)}$.

\section{Grouping of samples and Furcation repair pro- cedures}

Teeth were randomly divided into 3 experimental groups and control according to the perforation repair material then each group was further subdivided according to the evaluation period: 1 week, 1 month and 3 months 


\section{Group A: (Experimental tricalcium phosphate) $(n=30)^{(10)}$}

Powder (30\% Tri-Calcium Silicate, 30\% Di Calcium Silicate, 35\% Tri Calcium Phosphate and 5\% Bisthmus Oxide) and liquid (Di-sodium hydrogen phosphate $1 \%$ ) were mixed together until the desired consistency for a repair material was reached.

Group B: Biodentine (Septodont, Saint Maur des Fosse's, France) $(\mathrm{n}=30)$

Material was mixed according to the manufacturer's instructions

Group C: MTA (MTA Angelus, Brazil) (n=30)

Material was mixed according to the manufacturer's instructions

\section{Group D: (Control group) $(\mathrm{n}=6)$}

The perforation site was left open, without any repairing material or coronal seal, in order to verify the ability of the dye to penetrate the perforation.

Materials were condensed into the perforation site using condenser of the proper size until the perforation site was totally obliterated. The pulp chambers and the access cavities of the teeth were filled using Cavit (3M, ESPE, Germany) except controls; they were left open. All The teeth were then placed in physiologic saline in an incubator at $37^{\circ} \mathrm{C}$ during the different evaluation periods to simulate the clinical situation.

\section{The dye leakage procedures}

After each assessment period, teeth surfaces were coated with another layer of nail polish while avoiding perforation areas. Then, the teeth were immersed in $2 \%$ methylene blue dye for 72 -hours (11) at room temperature. Upon removal of the teeth from the dye, they were carefully washed with tap water and split buccolingually. Sections were examined and photographed for linear extent of dye penetration using digital camera (Canon, Japan)

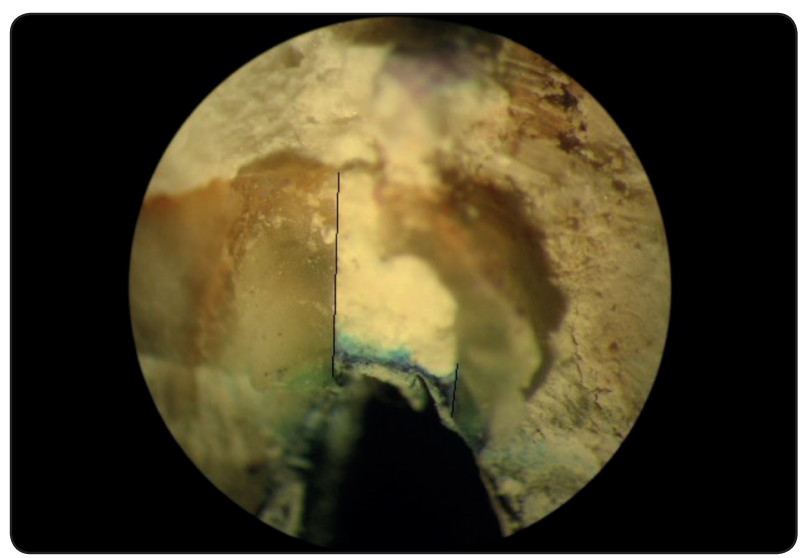

Fig. (1) Shows depth of dye penetration through perforation repair material using Canon digital camera connected to a Zeiss stereomicroscope (Technival 2) with magnification (25X).

connected to a Zeiss stereo microscope Technical 2 (ZEISS, Germany) with magnification (25X). In each sample, the highest point of dye penetration was measured and the percentage of linear extent of dye penetration from the apical end of the repair material to the pulp chamber floor in relation to the total length of perforation was calculated. The degree of dye penetration, in each microscopic field, at the area of perforation was measured using the image analysis software (Image ware, Image J 1.41, USA).

This equation was used to determine the dye penetration percent in relation to perforation walls in each section.:

Percentage of dye penetration $=\frac{\text { depth of penetration }}{\text { full length }} \times 100$

\section{Statistical Analysis}

Both mean and standard deviation were computed for each group in each test. Then, the normality of the data was evaluated using Kolmogorov-Smirnov and Shapiro-Wilk tests, but the results were not parametrically distributed. Kruskal Wallis test was utilized to differentiate between more than two groups in non-related samples. For comparison between two groups of unrelated samples, Mann 
Whitney test was advocated. Friedman assessment was utilized to differentiate between more than two groups in related sections whereas, Wilcoxon was utilized to correlate between 2-groups in associated samples. Significance level was adjusted at $\mathrm{P} \leq 0.05$. IBM ${ }^{\circledR}$ SPSS $®$ Statistics Version 20 for Windows was used.

\section{RESULTS}

A significant difference was present between the Control and the experimental groups. Values of mean dye penetration in experimental groups and controls in the current study showed that the highest dye penetration of all groups was in the control group followed by the Experimental material and MTA while Biodentine group showed least dye absorbance.

There was a significant difference between Biodentine and each of MTA and Experimental material groups whereas the sealing ability of Biodentine was best among the three materials tested. No statistically significant difference was found between MTA and (Experimental material) groups (Table 1).

Dye penetration increased in all of the groups tested with time, there was a statistically significant difference between the dye leakage percentages during the three evaluation periods for Biodentine group. While for both MTA and Experimental material no statistically significant difference between the dye leakage percentages during the three evaluation periods as shown in (Figure 2).

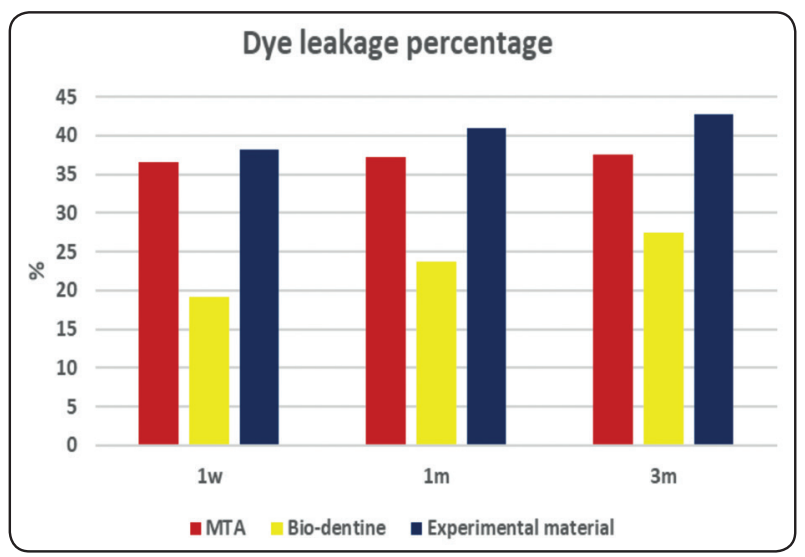

Fig. (2): Bar charts showing the mean values of the dye leakage percentage after 1 week, 1 month and 3 months.

\section{DISCUSSION}

Biocompatibility of the perforation repair material is the most important factor that affects how long the process will stay successful. Moreover, the repair material should be able to maintain acceptable sealing ability. Many methods such as dyes (India ink, methylene blue), chemical tracers, and radioactive isotopes were used to evaluate the sealing ability. Dye penetration procedure is the most popular method used to assess the sealing

TABLE (1): Dye leakage percentages for the MTA, Bio-dentine and Experimental material

\begin{tabular}{|c|c|c|c|c|c|c|c|}
\hline \multirow{3}{*}{ Variables } & \multicolumn{7}{|c|}{ Dye leakage \% } \\
\hline & \multicolumn{2}{|c|}{$1 w$} & \multicolumn{2}{|c|}{$1 \mathrm{~m}$} & \multicolumn{2}{|c|}{$3 \mathrm{~m}$} & \multirow{2}{*}{ p-value } \\
\hline & Mean & SD & Mean & SD & Mean & SD & \\
\hline Experimental material & 38.12 & 3.45 & 40.92 & 4.61 & 42.69 & 3.12 & $0.105 \mathrm{~ns}$ \\
\hline MTA & 36.60 & 1.12 & 37.24 & 1.37 & 37.55 & 5.34 & $0.472 \mathrm{~ns}$ \\
\hline Bio-dentine & 19.08 & 2.71 & 23.64 & 3.01 & 27.49 & 6.80 & $0.040 *$ \\
\hline p-value & \multicolumn{2}{|c|}{$0.025 *$} & \multicolumn{2}{|c|}{$0.015 *$} & \multicolumn{2}{|c|}{$0.013 *$} & \\
\hline
\end{tabular}

$*_{\text {significant }}(p<0.05) \quad n s ;$ non-significant $(p>0.05)$ 
ability of dental materials since it is an easy test to conduct and doesn't need complicated equipment. In addition, this technique is based on quantitative measurements of the passage of a liquid within these interfaces ${ }^{(12)}$.

Since it was reported by Niemann et al. ${ }^{(13)}$, in a study conducted on molar teeth, that lateral and accessory canals in the furcation area had patency allowing fluids to pass through them, then in this study, nail polish was used to paint the furcation region of all teeth.

Diameter of perforations was standardized by using a no. 2 round burs for their preparations, however, lengths of perforations remained an uncontrollable variable, as it depended on the thickness of dentin and cementum.

For by-passing the issue of unstandardized perforation length, dye penetration percentage in regard to perforation walls in each section was evaluated, using the image analysis software (Image J (Image ware, Image J 1.41, USA). A cotton pellet soaked in water was passively located in the furcation area to mock the moist clinical field.

MTA has been extensively used as perforation repair material due to its reported favorable sealing ability, biocompatibility and dentinogenic activity ${ }^{(14)}$. Biodentine is a bioactive ${ }^{(15)}$ dentine substitute specifically designed as a "dentine replacement" material. Tri-calcium silicate and Di-calcium silicate of the powder are considered as primary and secondary bulk materials whereas zirconium oxide is the radiopacifier. The liquid constitutes calcium chloride as the accelerator and a hydrosoluble polymer as water reducing agent ${ }^{(16)}$. Biodentine, is known to release Calcium when it's in solution just like MTA ${ }^{(17)}$.

Statistical analysis showed that the sealing ability of Biodentine was best among the three materials whereas sealing ability of the experimental tricalcium phosphate was similar to the MTA as perforation repair material. MTA encounters some problems during its clinical use, like; unease of handling, long setting time and liability to discolor the tooth ${ }^{(18)}$.

The sodium phosphate solution was used in order to allow the cement to harden more rapidly and improve the setting time; that is to decrease it. ${ }^{(19)}$ When studied earlier, setting time was found to be ${ }^{(20)} 19$ minutes (Kataia et al ${ }^{(21)} 2010$ ), Previous investigators ${ }^{(22,23)}$ brought the same conclusion that the use of high phosphate solution or a high $\mathrm{pH}$ solution would accelerate setting time.

The high sealing ability of Biodentine may be attributed to firm attachment of apatite crystals to the underlying dentine surface ${ }^{(24)}$ and excellent adaptability of this material to the underlying dentine due to nanostructure and small size of the forming gel ${ }^{(25)}$. Both experimental material and MTA showed good sealing ability and there was no statistically significant difference between them, this is also justified by the great similarity in the chemical composition between the two materials. Although not statistically significant but the sealing ability of both materials was found to decrease with time this is in accordance with other studies ${ }^{(26,27)}$.

\section{CONCLUSION}

The experimental tricalcium phosphate provides an easy handling and quick setting of repair material with comparable sealing ability to MTA. Further biological and cytotoxicity studies should be considered.

\section{REFERENCES}

1. Fuss Z, Trope M. Root perforations: Classification and treatment choices based on prognostic factors. Endod Dent Traumatol1996;12:255-64.

2. Seltzer S, Bender IB, Smith J, Freedman I, Nazimov H. Endodontic failures an analysis based on clinical, roentgenographic, and histologic findings. II. Oral Surg Oral Med Oral Pathol 1967;23:517-30. 
3. Tay et all 2007, Reyes-Carmona et al. 2009, Torabinejad \& parirokh 2010 .

4. About I: Bioactivity of BiodentineTM: A Ca3Si05based Dentine Substitute. Oral session, IADR Congress, Barcelona 2010.

5. Mir A, Misgar $\mathrm{OH}$, Farooq R,Purra AR, Ahanger FA.Comparison of Sealing Ability of Biodentine, Bioactive Bone Cement And MTA As Furcation Repair Materials. IOSR-JDMS 2017; 16:82-86.

6. Cardoso M, dos Anjos Pires M, Correlo V, Reis R, Paulo M, Viegas C. Biodentine for Furcation Perforation Repair: An Animal Study with Histological, Radiographic and Micro-Computed Tomographic Assessment. Iran Endod J. 2018;13(3):323-30.

7. Himel VT, Brady J, Weir J. Evaluation of repair of mechanical perforations of the pulp chamber floor using biodegradable tricalcium phosphate or calcium hydroxide. J Endod. 1985;11(4):161-65.

8. Alhadainy HA, Himel VT, Lee WB, Elbaghdady YM. Use of ahydroxylapatite-based material and calcium sulfate as artificial floorsto repair furcal perforations. Oral Surg Oral Med Oral PatholOralRadiol Endod. 1998; 68:723-729.

9. Alhadainy H, Abdalla A. Artificial floor technique used for the repair of furcation perforations. A microleakage study. J Endod. 1998;24(1):33-35.

10. Radwan MM, Khallaf M, and Kataia EM. Formulation and Characterization of a Calcium Silicate/Calcium Phosphate Root End Filling Material; Part II: Adaptability and InVivo Biocompatibility Study. RJPBCS 2016; 7:2474-2480.

11. Periera CL, Cenci MS, Demarco FF. Sealing ability of MTA, Super EBA, Vitremer and amalgam as root-end filling materials. Braz Oral Res. 2004;4(18):317-321.

12. Camps J, Pashley D. Reliability of the dye penetration studies, J Endod, 29, 2003, 592-4.

13. Niemann RW, Dickinson GL, Jackson CR, Wearden S, SkidmoreAE. Dye ingress in molars: furcation to chamber floor. J Endod.1993;19(6):293-6.

14. Sarkar NK, Caidedo R, Tirwik P, Moiseyeva R, Kawashima I. Physicochemical basis of the biologic properties of mineral trioxide aggregate, J Endod, 31, 2005, 97-100 .

15. Han L, Okiji T. Uptake of calcium and silicon released from calcium silicate-based endodontic materials into root canal dentine, Int Endod J, 44, 2011, 1081-7
16. Biodentine TM Active BiosilicateTechnology TM, Scientific file, Availablefrom:http://www.plandent.no/images/ Marketing/Infosenter/Bidentine\%20Scientific\%20File_ web_dokumentasjon.

17. Camilleri J, Kralj P, Veber M, Sinagra E. Characterization and analysis of acid-extractable and leached traced elements in dental cements, Int Endod J, 45, 2012, 737-43.

18. Baroudi K, Samir S. Sealing Ability of MTA Used in Perforation Repair of Permanent Teeth; Literature Review. Open Dent J. 2016;10:278-286.

19. Gbureck U, Barralet JE, Spatza K, Grover LM, and Thull R. Ionic modification of calcium phosphate cement viscosity. Part I: hypodermic 133.

20. International Standard ISO 6876:2012. DentistryRootCanalSealing Materials. 3rd ed. Vernier, Geneva, Switzerland: International Organization for StandardizationISO Central SecretariatChemin de Blandonnet; 2012.

21. Kataia EM, Ibrahim S, EL Hemaly, Zazou M, Lutfy R. Physical Properties and Adaptation of Synthesized Calcium Phosphate Cements: Comparative Study with New and Standard Root Canal Cements. PhD thesis. Faculty of Oral and Dental Medicine, Cairo University.

22. Takagi S, Chow LC, and Ishikawa K. Formation of hydroxyapatite in new calcium phosphate cements. Biomaterials 1998; 19: 1593-9.

23. Yoshikawa M, Terada Y, and Toda T. Setting time and sealing ability of $\alpha$ - tricalcium phosphate cement containing titanic oxide. J Osaka Dent Univ, 1998; 32: (2): 67-70.

24. Gjorgievska ES, Nicholson JW, Apostolska SM, et al. Interfacial properties of three different bioactive dentine substitutes, MicroscMicroanal, 19, 2013, 1450-7.

25. Koubi S, Elmerini H, Koubi G, Tassery H, Camps J. "Quantitative evaluation by glucose diffusion of microleakage in aged silicate-based open-sandwich restorations". International Journal of Dentistry, Vol. 2012, Article ID 105863, 6 Pages, 2012

26. Siqueira JF Jr, Isabela N. Roc, Ernani C. Abad, Castro AJ, Gahyva SA, Favier A. Ability of three root-end filling materials to prevent bacterial leakage. J Endod. 2001;27(11):673-5.

27. Al-Amoudi OA. In vitro study of the sealing ability of MTA versus Portland cement when used as an apical plug. MSc thesis. Faculty of Oral and Dental Medicine, Cairo University. 2009. 